\title{
Reduced IFN $\lambda 4$ activity is associated with improved HCV clearance and reduced expression of interferon-stimulated genes
}

Ewa Terczyńska-Dyla ${ }^{1, \star}$, Stephanie Bibert ${ }^{2, \star}$, Francois H.T. Duong ${ }^{3,4, \star}$, Ilona Krol ${ }^{3,4}$, Sanne Jørgensen ${ }^{1}$, Emilie Collinet $^{2}$, Zoltán Kutalik ${ }^{5,6}$, Vincent Aubert ${ }^{7}$, Andreas Cerny ${ }^{8}$, Laurent Kaiser ${ }^{9}$, Raffaele Malinverni ${ }^{10}$, Alessandra Mangia ${ }^{11}$, Darius Moradpour ${ }^{12}$, Beat Müllhaupt ${ }^{13}$, Francesco Negro ${ }^{14}$, Rosanna Santoro ${ }^{11}$, David Semela ${ }^{15}$, Nasser Semmo ${ }^{16}$, Swiss Hepatitis C Cohort Study Group $\ddagger$, Markus H. Heim³, ${ }^{3, \dagger}$, Pierre-Yves Bochud ${ }^{2, \dagger} \&$ Rune Hartmann ${ }^{1}$

Hepatitis $\mathrm{C}$ virus (HCV) infections are the major cause of chronic liver disease, cirrhosis and hepatocellular carcinoma worldwide. Both spontaneous and treatment-induced clearance of HCV depend on genetic variation within the interferon-lambda locus, but until now no clear causal relationship has been established. Here we demonstrate that an amino-acid substitution in the IFN $\lambda 4$ protein changing a proline at position 70 to a serine (P7OS) substantially alters its antiviral activity. Patients harbouring the impaired IFN $\lambda 4$-S70 variant display lower interferon-stimulated gene (ISG) expression levels, better treatment response rates and better spontaneous clearance rates, compared with patients coding for the fully active IFN $\lambda 4$-P70 variant. Altogether, these data provide evidence supporting a role for the active IFN $\lambda 4$ protein as the driver of high hepatic ISG expression as well as the cause of poor HCV clearance.

\footnotetext{
${ }^{1}$ Department of Molecular Biology and Genetics, Aarhus University, Aarhus DK-8000, Denmark. ${ }^{2}$ Infectious Diseases Service, Department of Medicine, University Hospital and University of Lausanne, Lausanne CH-1011, Switzerland. ${ }^{3}$ Department of Biomedicine, University of Basel, Basel CH-4031, Switzerland. ${ }^{4}$ Division of Gastroenterology and Hepatology, University Hospital Basel, Basel CH-4031, Switzerland. ${ }^{5}$ Institute of Social and Preventive Medicine, University Hospital (CHUV) and University of Lausanne, Lausanne 1010, Switzerland. ${ }^{6}$ Swiss Institute of Bioinformatics, Lausanne 1015, Switzerland. ${ }^{7}$ Service of Immunology and Allergology, Department of Medicine, University Hospital and University of Lausanne, Lausanne 1011, Switzerland. ${ }^{8}$ Fondazione Epatocentro Ticino, Sede Moncucco, Lugano 6900, Switzerland. ${ }^{9}$ Laboratory of Virology, Division of Infectious Diseases and Division of Laboratory Medicine, University Hospitals of Geneva and Medical School, University of Geneva, Geneva 1211, Switzerland. ${ }^{10}$ Pourtalès Hospital, Neuchâtel 2000, Switzerland. ${ }^{11}$ Liver Unit, Scientific Research Institute Casa Sollievo della Sofferenza, San Giovanni Rotondo 71013, Italy. ${ }^{12}$ Division of Gastroenterology and Hepatology, University Hospital and University of Lausanne, Lausanne CH-1011, Switzerland. ${ }^{13}$ Division of Gastroenterology and Hepatology, University Hospital of Zurich, Zurich 8091, Switzerland. ${ }^{14}$ Division of Clinical Pathology and Division of Gastroenterology and Hepatology, University Hospitals, Geneva 1211, Switzerland. ${ }^{15}$ Division of Gastroenterology, Canton Hospital, St Gallen CH-9007, Switzerland. ${ }^{16}$ Service of Hepatology, Department of Clinical Research, University of Bern, Bern CH-3010, Switzerland. * These authors contributed equally to this work. $\dagger$ These authors jointly supervised this work. $\ddagger$ The members of the Swiss Hepatitis C Cohort Study (SCCS) appear at the end of the paper. Correspondence and requests for materials should be addressed to M.H.H. (email: Markus.Heim@unibas.ch) or to P.-Y.B. (email: Pierre-Yves.Bochud@chuv.ch) or to R.H. (email: RH@mbg.au.dk)
} 
$\mathrm{H}$ epatitis $\mathrm{C}$ virus (HCV) infections are the major cause of chronic liver disease, cirrhosis and hepatocellular carcinoma worldwide ${ }^{1}$. HCV is parenterally transmitted, mainly due to injection drug use, unsafe transfusions and therapeutic injections. In $70-80 \%$ of those infected, the virus persists and the infection becomes chronic, and eventually requires antiviral therapy. During the past 25 years, recombinant interferon-alpha (IFN $\alpha$ ) has been an essential component of HCV treatment ${ }^{2}$, however, the recent availability of potent direct-acting antiviral agents (DAA) is changing the therapeutic options.

The overall sustained virological response (SVR) rate achieved using a combination of pegylated IFN $\alpha$ (pegIFN $\alpha$ ) and ribavirin is only about $50 \%$, and the prognosis for individual patients strongly depends on their genetic background. In particular, the genetic variation within the IFNL3 (IL28B) locus is crucial in determining the treatment outcome ${ }^{3-6}$. Furthermore, the response to IFN-based therapy depends strongly on the expression of IFN-stimulated genes (ISGs) in patients before the initiation of treatment ${ }^{7-9}$ and variations near the IFNL3 (IL28B) locus correlate with the ISG induction in the liver ${ }^{10-12}$. Somewhat paradoxically, patients showing increased pretreatment expression of ISG in the liver respond poorly to pegIFN $\alpha /$ ribavirin therapy ${ }^{7-9}$. However, the mechanism responsible for driving ISG induction in the $\mathrm{HCV}$-infected liver has remained elusive. Roughly, a quarter of $\mathrm{HCV}$-infected individuals clear the virus spontaneously, this largely happens during the acute phase of the infection. Somewhat surprisingly, the genetic variations near the IFNL3 (IL28B) locus are also strongly associated with spontaneous clearance of $\mathrm{HCV}$ infection ${ }^{13,14}$. That points towards a pivotal role for IFN $\lambda \mathrm{s}$ in $\mathrm{HCV}$ clearance and a possible influence of IFN $\lambda$ s on the adaptive immune response that is generated during the acute phase of HCV infection.

A recent key discovery identified the IFNL4 gene and showed that a polymorphism ( $r s 368234815)$, which causes the disruption of the IFNL4 reading frame, correlates with a substantially better treatment response ${ }^{15,16}$ as well as better spontaneous clearance rate ${ }^{13}$. Thus, patients having a nonfunctional IFNL4 gene are more likely to clear the HCV infection. This suggests that the production of IFN $\lambda 4$ in carriers of the ancestral $r s 368234815 \Delta \mathrm{G}$ allele is responsible for the transcriptional activation of ISGs, thus providing a molecular link between the genotype and clearance of HCV. This hypothesis is supported by the recent finding that recombinant IFN $\lambda 4$ protein strongly stimulates Jak-STAT signalling and ISG induction through binding to the IFN $\lambda$ receptor ${ }^{17}$. Here we describe a strong link between a reduced antiviral activity of the IFN $\lambda 4$ protein and a reduced hepatic ISG expression as well as an improved HCV clearance.

\section{Results}

Substitution at position 70 impairs the activity of IFN $\lambda 4$. Three non-synonymous single-nucleotide polymorphisms (SNPs) in the IFNL4 gene were previously described but not characterized ${ }^{15,18}$, those being C17Y (rs73555604), R60P (rs142981501) and P70S (rs117648444). We noted that a P70S substitution (rs117648444) could potentially alter the activity of the protein, in addition this has a minor allele frequency (MAF) in Caucasian of 0.11 (HapMap), whereas the two other have 0 and 0.025 , respectively. Proline 70 is located outside of the assumed receptor binding site ${ }^{19,20}$, but structural modelling suggests that P70 is important for the stability of the IFN $\lambda 4$ protein ${ }^{17}$. In the following, the two variants of IFN $\lambda 4$ will be denoted as IFN $\lambda 4$-P70 and IFN $\lambda 4$-S70, respectively.
We expressed the mature form of both variants in Escherichia coli, purified them (Fig. 1a) and tested their activity. The IFN $\lambda 4$ S70 variant induced significantly lower levels of both $M X 1$ and IFIT1 transcription in human hepatocellular carcinoma cells (HepG2; Fig. 1b). Next, a dose-response experiment was performed in human embryonic kidney cells (HEK293) transfected with an IFN-responsive firefly luciferase reporter construct, a Renilla luciferase construct for normalization and a construct expressing the IFN $\lambda \mathrm{R} 1$ receptor chain (Fig. 1c). We obtained half-maximal effective concentration $\left(\mathrm{EC}_{50}\right)$ values of

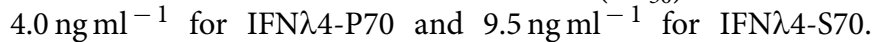
This difference was significant using $99 \%$ confidence intervals (Supplementary Table 1). Finally, we performed an antiviral assay using encephalomyocarditis virus (EMCV) infection in HepG2 cells (Fig. 1d; Supplementary Table 1). Here we saw a significant shift in the $\left(\mathrm{EC}_{50}\right)$ values from $0.2 \mathrm{ng} \mathrm{ml}^{-1}$ for the IFN $\lambda 4$-P70 to $1.4 \mathrm{ng} \mathrm{ml}^{-1}$ for IFN $\lambda 4$-S70. Thus, the IFN $\lambda 4$-S70 variant is substantially less active than the IFN $\lambda 4$-P70 variant.

$r s 117648444^{\mathrm{A}}$ is an independent predictor of $\mathrm{HCV}$ clearance. If the active IFN $\lambda 4$ protein is the causative agent responsible for the poor HCV clearance in patients, then one would expect the P70S substitution ( $r s 117648444)$ to influence the ability of patients to clear HCV. Thus, we evaluated the role of the $r s 117648444^{\mathrm{A}}$ $(\mathrm{IFN} \lambda 4-\mathrm{S} 70, \mathrm{MAF}=0.11)$ and $r s 368234815^{\Delta \mathrm{G}}$ (expressing IFN $\lambda 4$, $\mathrm{MAF}=0.35)$ polymorphisms in $\mathrm{HCV}$ clearance in a cohort of 574 chronically infected patients who underwent pegIFN $\alpha /$ ribavirin treatment, as well as in 122 patients with spontaneous clearance (Table 1; Supplementary Tables 2 and 3). We analysed the independent contribution of each polymorphism, by including both of them in the same logistic regression model. As expected, $r s 368234815^{\Delta \mathrm{G}}$ was associated with a lower SVR rate (odds ratio $(\mathrm{OR})=0.23,95 \%$ confidence interval $(\mathrm{CI})$ 0.15-0.35, $P=1.5 \mathrm{E}-11$, dominant mode of inheritance), while $r s 117648444^{\mathrm{A}}$ (IFN $\lambda 4$-S70) was associated with a higher SVR rate $(\mathrm{OR}=1.66$, $95 \%$ CI $1.08-2.55, P=0.02$ ). Thus, both $r s 117648444^{\mathrm{A}}$ and $r s 368234815^{\Delta \mathrm{G}}$ are independent predictors of treatment-induced HCV clearance. A similar trend was observed for spontaneous clearance $(\mathrm{OR}=0.21, \quad 95 \% \quad \mathrm{CI} \quad 0.13-0.34, \quad P=4.4 \mathrm{E}-10$ for $r s 368234815^{\Delta \mathrm{G}} ; \mathrm{OR}=1.63,95 \%$ CI $0.88-3.02, \quad P=0.12$ for rs 117648444 ${ }^{\mathrm{A}}$; Table 1).

Stratification of patients according to IFN $\lambda 4$ functionality. A haplotype analysis was performed to understand the co-inheritance pattern of $r s 368234815$ and $r s 117648444$, which would help to clarify the role of the activity of IFN $\lambda 4$ in HCV clearance. This analysis showed that $95 \%$ of the chromosomes are composed of three main haplotypes: TT G $(65 \%)$, which is predicted not to produce IFN $\lambda 4, \Delta \mathrm{G} \mathrm{G}(22 \%)$, which is predicted to produce the IFN $\lambda 4-\mathrm{P} 70$ variant, and $\Delta \mathrm{G} \mathrm{A}$, which is predicted to express the IFN $\lambda 4$-S70 variant (11\%; Supplementary Table 4). The frequency distribution in our study population is in accordance with previously published data ${ }^{15,18}$. The fourth haplotype TT A, which does not express IFN $\lambda 4$, was found in only $0.5 \%$ of the chromosomes, and is grouped with the TT G haplotype in the subsequent analyses. The $\Delta \mathrm{G} G$ haplotype (IFN $\lambda 4-\mathrm{P} 70$, $R^{2}=0.74$ ) was in linkage disequilibrium (LD) with the SNP rs8099917, which emerged as the top hit for spontaneous or treatment-induced HCV clearance in three out of four initial hepatitis C genome-wide association studies (GWAS; Table 2).

The haplotypic combinations produced eight diplotypes (Table 3), which can be classified into three main functional groups according to their ability to produce IFN $\lambda 4-\mathrm{P} 70$ or IFN $\lambda 4$-S70. The first group consists of patients not producing IFN $\lambda 4(N=283)$. The second group contains diplotypes that 
a

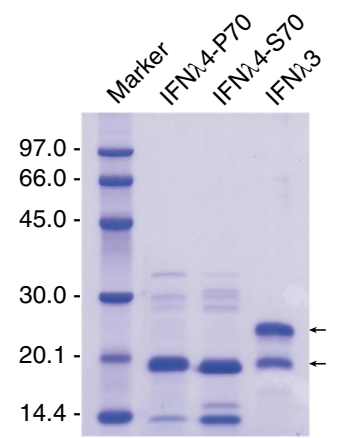

c

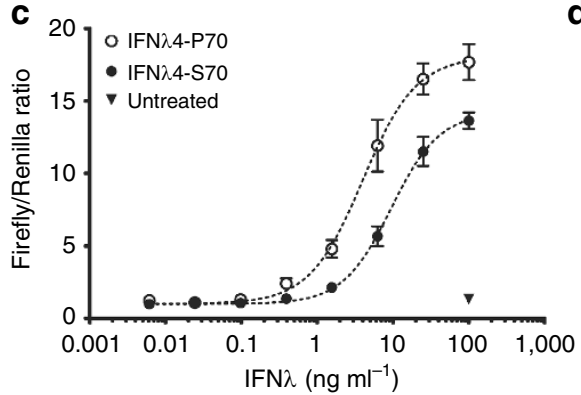

b

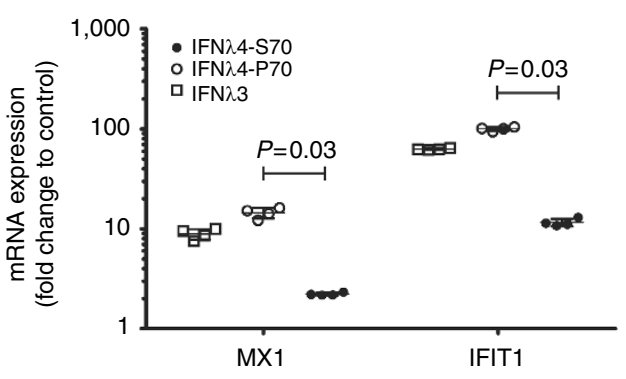

d

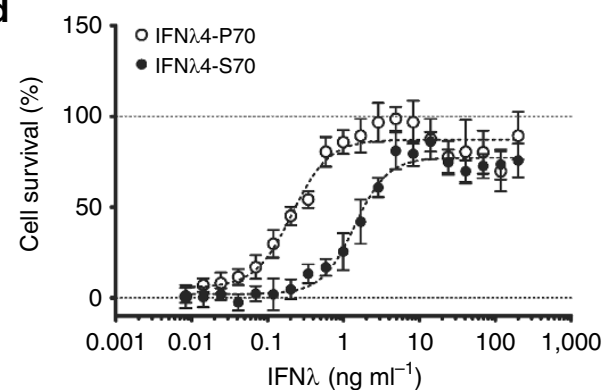

Figure 1 | Activity of recombinant human IFN $\mathbf{4}$-P70 and IFN $\mathbf{\lambda}$ 4-S70 variants. Data represent the results of multiple experiments with similar results. (a) A coomassie-stained $12 \%$ SDS-polyacrylamide gel electrophoresis of the purified proteins. Cleavage of the $\mathrm{N}$-terminal His-Tag was only partial for IFN $\lambda 3$, hence the double band. The upper arrow indicates the migration of the IFN $\lambda 3$-His-tag fusion protein; the lower arrow indicates migration of the mature IFN $\lambda 3 / \lambda 4$ proteins. (b) HepG2 cells were treated with $1 \mathrm{ng} \mathrm{ml}{ }^{-1}$ of IFN $\lambda 3$, IFN $\lambda 4$-P70 or IFN $\lambda 4$-S70. After $4 \mathrm{~h}$, the mRNA levels of the ISGs IFIT1 and MX1 were quantified by real-time quantitative PCR. Experiments were performed in quadruplicates; replicates, mean and s.d. are plotted.

The $P$ value for IFN $\lambda 4$-P70 versus IFN $\lambda 4$-S70 is shown (Mann-Whitney test). (c) The activity of IFN 4 4-S70 was tested and compared with IFN $\lambda 4$-P70 in HEK293 cells, which were transfected with the pEF2 vector containing IFN $\lambda$ R1, as well as Renilla and firefly luciferase reporters $24 \mathrm{~h}$ before IFN treatment. The firefly construct is IFN inducible, whereas the Renilla construct is constitutively expressed. Dose responses of IFNs were performed in quadruplicates. Mean and s.d. are shown. (d) HepG2 cells were treated with IFN $24-S 70$ or IFN $24-P 70$ for $24 \mathrm{~h}$ and subsequently infected with EMCV. Cell survival was analysed using the conversion of MTT to formazan in living cells by measurement of the $A_{600}$. Data are presented as a percentage of a non-infected cell control. Error bars depict s.d. of 10 replicates.

exclusively code for IFN $\lambda 4-S 70 \quad(N=114)$. The third group consists of those that give rise to the fully functional IFN $\lambda 4-\mathrm{P} 70$ $(N=299)$. This group is composed of 270 samples with the TT $\mathrm{G} / \Delta \mathrm{G} G$ and the $\Delta \mathrm{G} G / \Delta \mathrm{G} G$ diplotype producing only the IFN $\lambda 4$-P70 variant, and 29 samples with the $\Delta G A / \Delta G G$ diplotype producing both variants of IFN $\lambda 4$.

IFN $\lambda$ 4-S70 associates with a better clearance rate of $\mathrm{HCV}$. We analysed the response to treatment in patients producing IFN $\lambda 4$ S70 alone and those producing IFN $\lambda 4$-P70 alone grouped with IFNL4-P70/S70 compared with those not producing IFN $\lambda 4$. Patients not producing IFN $\lambda 4$ had an overall SVR rate of $81 \%$ after treatment with pegIFN $\alpha /$ ribavirin compared with only $47 \%$ among those who produced the IFN $\lambda 4-\mathrm{P} 70$ or $\mathrm{P} 70 / \mathrm{S} 70$ variants $(\mathrm{OR}=0.21,95 \%$ CI $0.14-0.33, P=8.3 \mathrm{E}-13$; Table 1; Fig. 2a). We then excluded patients not producing IFN $\lambda 4$ to specifically assess the role of the P70S substitution. Interestingly, patients who produced exclusively the IFN $\lambda 4$-S70 had a much higher response rate $(69 \%)$ compared with those producing the IFN $\lambda 4-\mathrm{P} 70$ or P70/ S70 variants $(\mathrm{OR}=2.52,95 \% \mathrm{CI} 1.55-4.13, P=0.0002)$. This clearly shows that the chance of clearing the virus is highest in the group of patients not expressing IFN $\lambda 4$, slightly lower in those expressing the impaired IFN $\lambda 4$-S70 variant and the worst in those expressing the fully active IFN $\lambda 4$-P70 form. The differences in SVR rates between all three groups were significant. This agrees well with the biochemical data, which show that IFN $\lambda 4$-S70 has an impaired but still clearly measurable activity. These associations were still significant in a multivariate model after adjustment for relevant covariates (Table 1). The association was similar, although less significant, when the analysis was restricted to patients infected with HCV genotype 1 (Supplementary Table 5).

Roughly, a quarter of all $\mathrm{HCV}$-infected individuals spontaneously clear the HCV infection, mainly during the acute phase of the infection by mounting an efficient immune response to the virus $^{21}$. We investigated whether the level of IFN $\lambda 4$ activity was associated with the spontaneous clearance. Similarly to what was observed for the treatment-induced clearance, the proportion of patients with spontaneous clearance was $29 \%$ among patients unable to produce IFN $\lambda 4,7 \%$ among those producing the IFN $\lambda 4$ P70 or P70/S70 variants and $15 \%$ among those producing exclusively IFN $\lambda 4$-S70 (Table 1; Fig. 2b).

IFN $\lambda$ 4-P70 is associated with high ISG expression. We have previously shown that the quantification of the expression of four ISGs (IFI27, ISG15, RSAD2 and HTATIP2) in liver biopsy samples allows one to predict the response to pegIFN $\alpha /$ ribavirin treatment ${ }^{10}$. This 4-gene classifier was obtained through a multivariate analysis using decision tree ensembles in the form of a random forest classifier and is expressed as the probability of obtaining a sustained virological response (pSVR). The pSVR value contains the collective information from the four genes measured and is inversely correlated with ISG expression in the liver ${ }^{10}$. In the present work, the 4 -gene classifier was used as a surrogate marker for the overall ISG expression in 104 liver biopsies from patients with chronic hepatitis $\mathrm{C}$. The biopsies were then genotyped for the IFN $\lambda 4$ variants and classified into the three groups corresponding to the genomic analysis described above. In an analysis including all viral genotypes, the median 
Table 1 | Association between IFNL4 rs368234815 and rs117648444 polymorphisms and spontaneous and treatment-induced HCV clearance.

\begin{tabular}{|c|c|c|c|c|c|c|c|}
\hline \multirow{2}{*}{ Response to treatment $(N=574)$} & \multirow[t]{2}{*}{ NR } & \multirow[t]{2}{*}{ SVR } & \multirow{2}{*}{ Proportion SVR } & \multicolumn{2}{|c|}{ Univariate models } & \multicolumn{2}{|c|}{ Multivariate models $^{\star}$} \\
\hline & & & & OR $(95 \% \mathrm{Cl})$ & $P$ value & OR $(95 \% \mathrm{Cl})$ & $P$ value \\
\hline \multicolumn{8}{|c|}{ Individual contribution of both polymorphisms } \\
\hline $\begin{array}{rr}r s 368234815 & T T /-G \text { or }-G /-G \text { vers } \\
r s 117648444 & G / A \text { or } A / A \text { versus }\end{array}$ & $\begin{array}{l}\text { TT/TT } \\
\text { G }\end{array}$ & & & $\begin{array}{l}0.23(0.15-0.35) \\
1.66(1.08-2.55)\end{array}$ & $\begin{array}{l}1.5 \mathrm{E}-11 \\
0.02\end{array}$ & $\begin{array}{l}0.16(0.09-0.28) \\
2.35(1.31-4.26)\end{array}$ & $\begin{array}{c}3.2 \mathrm{E}-10 \\
0.004\end{array}$ \\
\hline
\end{tabular}

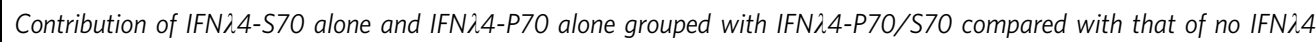
No IFN $\lambda 4$

IFN $\lambda 4-570$ alone

IFN $\lambda 4-P 70$ and P70/S70

$\begin{array}{rrr}39 & 161 & 0.81 \\ 30 & 67 & 0.69 \\ 147 & 130 & 0.47\end{array}$
Reference $0.54(0.31-0.94)$

$0.21(0.14-0.33)$

0.03

$8.3 E-13$

Reference

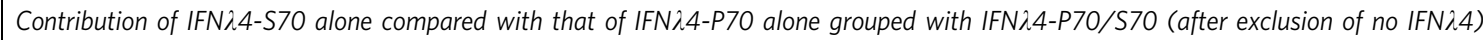

IFN $\lambda 4$-S70 a

$\begin{array}{lll}30 & 67 & 0.69\end{array}$

2.52 (1.55-4.13)

0.0002

Reference

$0.61(0.29-1.28)$

$0.14(0.08-0.26)$

IFN $\lambda 4-P 70$ and P70/S70

$147 \quad 130$

0.47

OR $(95 \% \mathrm{Cl})$

$4.22(2.10-8.51)$

5.5E-05

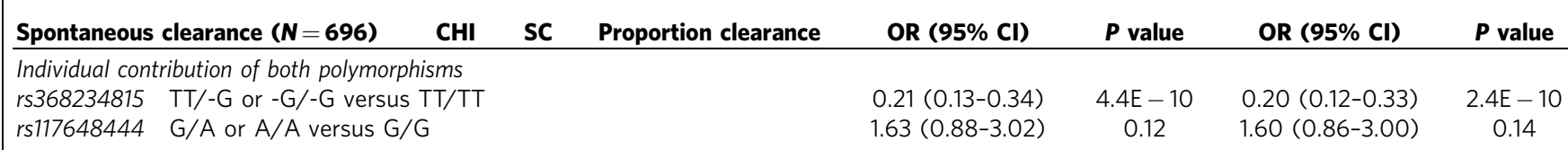

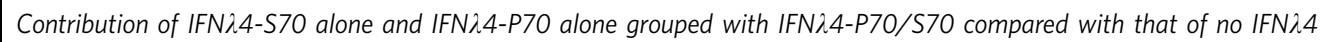

No IFN $\lambda 4$

$200 \quad 83 \quad 0.29$

0.15

Reference

IFN $\lambda$ 4-S70 alone

$97 \quad 17$

0.07

$0.42(0.24-0.75)$

Reference

IFN $\lambda 4$-P70 and P70/S70

$277 \quad 22$

$0.19(0.12-0.32) \quad 1.3 \mathrm{E}-10$

$0.41(0.23-0.74)$

0.003

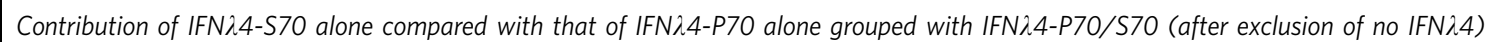

\begin{tabular}{lllllcc} 
IFN $\lambda$ 4-S70 alone & 97 & 17 & 0.15 & $2.21(1.12-4.33)$ & 0.02 & $2.27(1.13-4.54)$ \\
IFN $\lambda 4-P 70$ and P70/S70 & 277 & 22 & 0.07 & Reference & 0.02 \\
\hline
\end{tabular}

$\mathrm{Cl}$, confidence interval; $\mathrm{CHI}$, chronic infection; NR, non-response to treatment; OR, odds ratio; SC, spontaneous clearance; SVR, sustained virological response.

${ }^{\star}$ Multivariate models for spontaneous clearance are adjusted for age, sex and HCV risk. Multivariate models for response to treatment are adjusted for age, sex, fibrosis stage and viral genotype.

Multivariate models include a smaller number of treated patients $(N=404)$ due to missing covariates.

Table 2 | Linkage disequilibrium between different IFNL4 polymorphisms.

\begin{tabular}{|c|c|c|c|c|c|c|c|c|}
\hline & rs8099917 & rs12979860 & rs368234815 & rs117648444 & TT G (no IFN $\lambda 4)$ & $\begin{array}{c}\Delta G \text { G (IFN } \lambda \\
\text { 4-P70) }\end{array}$ & $\begin{array}{c}\Delta G A(\text { IFN } \lambda \\
\text { 4-S70) }\end{array}$ & $\begin{array}{c}\text { TT A } \\
\text { (no IFN } \lambda 4)\end{array}$ \\
\hline rs8099917 & 0.23 & & & & & & & \\
\hline rs12979860 & 0.45 & 0.37 & & & & & & \\
\hline rs368234815 & 0.44 & 0.92 & 0.35 & & & & & \\
\hline rs117648444 & 0.03 & 0.21 & 0.19 & 0.11 & & & & \\
\hline TT G (no IFN $\lambda 4)$ & 0.43 & 0.93 & 0.98 & 0.23 & 0.65 & & & \\
\hline$\Delta G \mathrm{G}(\mathrm{IFN} \lambda 4-\mathrm{P} 70)$ & 0.74 & 0.53 & 0.60 & 0.04 & 0.59 & 0.24 & & \\
\hline$\Delta G \mathrm{~A}(\mathrm{IFN} \lambda 4-\mathrm{S} 70)$ & 0.03 & 0.21 & 0.22 & 0.95 & 0.22 & 0.04 & 0.11 & \\
\hline TT A (no IFN $\lambda 4)$ & 0.00 & 0.00 & 0.00 & 0.04 & 0.01 & 0.01 & 0.00 & 0.01 \\
\hline
\end{tabular}

pSVR was $85 \%$ in group 1 (no IFN $\lambda 4, N=39$ ), $72 \%$ in group 2 (functionally defective IFN $\lambda 4-S 70, N=12$ ) and $46 \%$ in group 3 (fully functional IFN $\lambda 4-\mathrm{P} 70, N=53$ ). The difference between groups 1 and 3 , but not between 1 and 2, was statistically significant (Fig. 3a). The results did not change when the analysis was restricted to patients infected with HCV genotype 1 (Fig. 3b). Thus, there is a clear correlation between the activity of the IFN $\lambda 4$ protein and the hepatic ISG expression level.

\section{Discussion}

Because IFN $\lambda 4$-P70, but not the functionally defective IFN $\lambda 4$ S70, is associated with a strong induction of ISG expression (and therefore a low pSVR), we conclude that IFN $\lambda 4$ is the major component of the molecular mechanisms responsible for hepatic ISG expression in patients with $\mathrm{HCV}$ infections. Sustained signalling through the IFN $\lambda$ receptor can inhibit subsequent responses to type I IFN through the induction of the USP18 protein $^{22,23}$. Therefore, prolonged IFN $\lambda$ signalling caused by the IFN $\lambda 4$ protein is obviously inhibitory to subsequent treatment with pegIFN $\alpha /$ ribavirin. Interestingly, previous work has observed an association between both SNP rs12979860 (ref. 14) (situated in the IFNL4 intronic region) and rs368234815 (ref. 13) (the $\Delta \mathrm{G}$ allele determining IFN $\lambda 4$ expression) and the rate of spontaneous clearance of $\mathrm{HCV}$. We observed a clear correlation between the activity of IFN $\lambda 4$ and the spontaneous clearance rate. Patients producing the impaired IFN $\lambda 4-$ S70 had a $15 \%$ chance of spontaneous clearance compared with a $7 \%$ clearance rate among 
Table 3 | IFNL4 haplotype combination and associated clearance phenotypes.

\begin{tabular}{|c|c|c|c|c|c|c|c|c|c|c|}
\hline \multirow[t]{2}{*}{ Diplotype $^{\star}$} & \multirow[t]{2}{*}{$\mathbf{N}$} & \multirow[t]{2}{*}{ Proportion of patients } & \multicolumn{2}{|c|}{ IFN $\lambda 4$ production } & \multicolumn{2}{|c|}{ SVR } & \multirow[t]{2}{*}{ \% SVR } & \multirow[t]{2}{*}{ CHI } & \multirow[t]{2}{*}{ SC } & \multirow[t]{2}{*}{$\%$ SC } \\
\hline & & & 70P & $70 S$ & - & + & & & & \\
\hline TT G/TT G & 277 & 0.398 & - & - & 39 & 156 & 0.800 & 195 & 82 & 0.296 \\
\hline $\mathrm{TT} \mathrm{G} / \Delta \mathrm{G} \mathrm{G}$ & 231 & 0.332 & + & - & 111 & 105 & 0.486 & 216 & 15 & 0.065 \\
\hline $\mathrm{TT} \mathrm{G} / \Delta \mathrm{G} A$ & 110 & 0.158 & - & + & 30 & 64 & 0.681 & 94 & 16 & 0.146 \\
\hline$\Delta G \mathrm{G} / \Delta \mathrm{G} \mathrm{G}$ & 39 & 0.056 & + & - & 17 & 17 & 0.500 & 34 & 5 & 0.128 \\
\hline$\Delta G A / \Delta G G$ & 29 & 0.042 & + & + & 19 & 8 & 0.296 & 27 & 2 & 0.069 \\
\hline TT G/TT A & 5 & 0.007 & - & - & 0 & 4 & 1.000 & 4 & 1 & 0.200 \\
\hline$\Delta G A / \Delta G A$ & 4 & 0.006 & - & + & 0 & 3 & 1.000 & 3 & 1 & 0.250 \\
\hline TT A/TT A & 1 & 0.001 & - & - & 0 & 1 & NA & 1 & 0 & NA \\
\hline
\end{tabular}
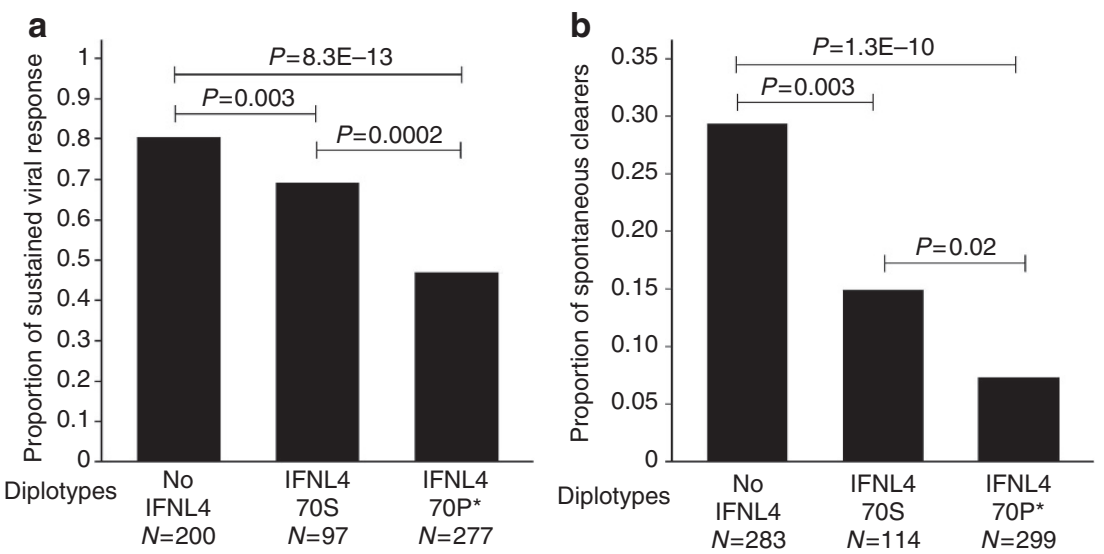

Figure 2 | Association of IFNL4 rs368234815 and rs117648444 polymorphisms and their diplotypes with treatment-induced and spontaneous HCV clearance. Patients were divided into three groups based on their diplotype: those predicted not to produce IFN $\lambda 4$, those predicted to produce IFN $\lambda 4-\mathrm{S} 70$ and those predicted to produce IFN $\lambda 4$-P70. The latter group included 29 patients $\left(^{*}\right)$ with a diplotype encoding both IFN $24-P 70$ and IFN $\lambda 4-S 70$. Data represent the proportion of patients infected with all HCV genotypes, who achieved (a) sustained virological response after treatment with pegIFN $\alpha$ / ribavirin or (b) spontaneous clearance. $P$ values were obtained by logistic regression. $N=$ number of patients in a given group.

a

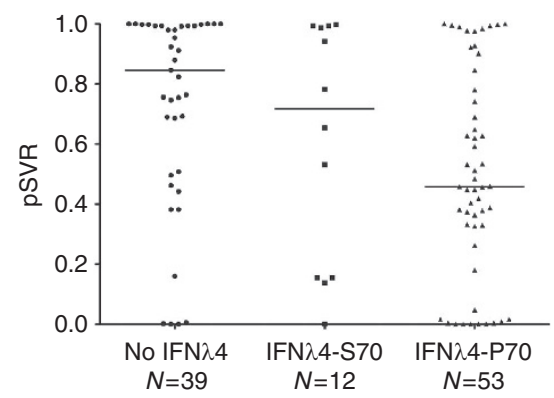

b

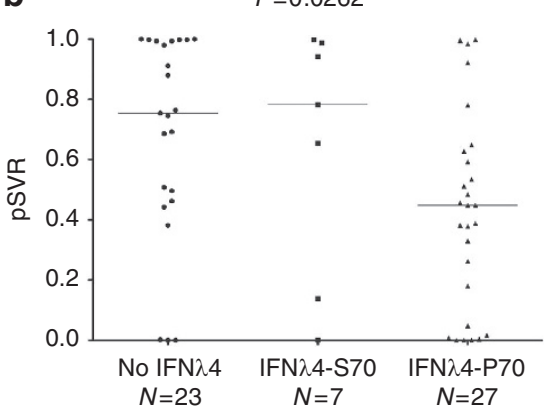

Figure 3 | Association of IFNL4 genotype with ISG expression in liver biopsies. Overall ISG expression in liver biopsies was assessed with the 4-gene classifier calculating the probability of a sustained virological response for pegIFN $\alpha$ /ribavirin treatments. A high pSVR corresponds to low overall ISGs expression in the liver and vice versa. The biopsies were grouped according to their IFNL4 diplotype as indicated. Shown are results including (a) all HCV genotypes or (b) HCV genotype 1 only. Statistical analysis with Kruskal-Wallis revealed significant overall differences of the median pSVR of the three groups for all genotypes and for genotype 1 only, with $P=0.0058$ and $P=0.026$, respectively. Between groups, only the groups 'no IFN $\lambda 4$ ' and 'IFN $\lambda 4$ P70' were significantly different from each other (Dunn's multiple comparison test). $N$ =number of samples per group.

patient coding for the active IFN $\lambda 4$-P70 variant. Among patients homozygous for the rs368234815 TT allele (major allele, causing a IFNL4 frame shift mutation), the clearance rate was increased to $29 \%$. As the majority of spontaneous clearance occurs during the acute phase of $\mathrm{HCV}$ infection, the IFN $\lambda 4$ protein must influence the early phase of an HCV infection in a way that is favouring development of a chronic infection and thus impairing spontaneous clearance. We speculate that the IFN $\lambda 4$ protein can influence adaptive immunity, which is established during the acute phase, and that this is a long-lasting influence. Our data link the active form of IFN $\lambda 4$ (IFN $\lambda 4$-P70) to high ISG expression and thus to low level of viral replication, which somewhat counterintuitively leads to poor treatment response. We suggest that IFN $\lambda 4$-P70-driven ISG expression causes lower level of viral 
replication, which in turn leads to an inefficient adaptive immune response, and that this is the base of poor HCV clearance. This is supported by a recent study showing an association between the $r s 368234815 \Delta \mathrm{G}$ allele (IFN $\lambda 4$ expression) and activity level of $\mathrm{T}$ cells ${ }^{24}$. Furthermore, our findings are supported by a recent paper, showing that patients carrying the IFN $\lambda 4$-S70-coding allele exhibit a faster decline in HCV RNA levels at week 4 of treatment ${ }^{25}$.

Combining rs368234815 and rs117648444 polymorphisms, it is possible to stratify patients into three groups, with the highest cure rates in patients not producing IFN $\lambda 4$, intermediate cure rates among those producing the IFN $\lambda 4$-S70 variant and the lowest cure rates among those producing the IFN $\lambda 4$-P70 variant. Furthermore, there is an excellent agreement between the predicted response rate (pSVR) obtained by measuring ISG expression in liver biopsies and the experientially observed SVR, once patients are stratified according to the three groups defined above. In our cohort, $26 \%$ of the patients being able to express IFN $\lambda 4$ harbour only the IFN $\lambda 4$-S70 variant, and thus have a prognosis that is almost as good as those not producing IFN $\lambda 4$. Thus, by combining both polymorphisms, it is possible to substantially improve the accuracy of the predicted response rate before treatment. In addition, we can clarify a discrepancy that has puzzled the field. The $r s 368234815$ polymorphism that determines the ability to express IFN $\lambda 4$ is not in LD with rs8099917, which was found as a good SVR predictor in three out of four of the initial GWAS studies ${ }^{3-6}$. This was unexpected and did initially argue against the IFN $\lambda 4$ protein as the causative agent; however, it is now clear that the minor allele of $r s 8099917$ specifically tags the haplotype producing the IFN $\lambda 4$-P70 variant, which we have shown to be associated with the poorest clearance rates.

One may argue that the use of genetic markers may become obsolete with the advent of direct-acting antiviral agents (DAAs), due to their high cure rates ${ }^{26-28}$. Nevertheless, the role of IFNL3/ IFNL4 polymorphisms has been convincingly demonstrated in the first generation DAA treatment regimes. The SOUND-C2 trial assessing the combination of faldaprevir and deleobuvir with or without ribavirin showed a significantly higher SVR rates for patients homozygous for the favourable allele of $r s 12979860$ (CC), clearly demonstrating a role for IFNL genotype in IFN-free DAA treatment regimens ${ }^{29}$. Thus, in mechanistic term, the role of the IFNL genotype is not restricted to IFN-based therapies, but do influence DAA-based treatments. However, recent development in DAA combination therapies using drugs with a substantially improved potency has pushed SVR above $90 \%$ for all patients, regardless of their IFNL genotype. With cure rates, this high knowledge of the genotype will have little impact on patients' choices of treatment. However, there are several clinical as well as scientific reasons to pursue a deeper understanding of how IFNL genotype impact viral clearance. Several recent studies show that rs368234815 influences the response kinetics to $\mathrm{DAA}^{28,30}$; however, the role of $r s 117648444$ in DAA treatment has not yet been investigated. This suggests that patients not expressing IFN $\lambda 4$ can receive a shortened DAA treatment and possibly also reduced doses. There will be both obvious benefits for patients and substantial cost reduction by a targeted shortening of therapy in patients with favourable IFNL genotype. Our study clearly shows that the decision should be based on a dual genotyping of rs117648444 and rs368234815.

The expression of active IFN $\lambda 4$ influences both IFN-based and IFN-free treatment of chronic HCV infections, as well as strongly influences the spontaneous clearance rate. These observations point towards a fundamental role of active IFN $\lambda 4$ in facilitating and maintaining a chronic $\mathrm{HCV}$ infection. A recent study showed that $r s 368234815$ also influenced susceptibility to cytomegalovirus retinitis among $\mathrm{HIV}$-infected patients ${ }^{31}$, suggesting that the IFN $\lambda 4$ protein might play detrimental role in several different chronic viral infections. Thus, we believe that the activity of IFN $\lambda 4$ could play an important role in multiple chronic viral infections by a mechanism similar to that observed in hepatitis $\mathrm{C}$.

\section{Methods}

Protein expression and purification. BL21 (DE3) E. coli cells transformed with the plasmids were grown at $37^{\circ} \mathrm{C}$ in Luria Bertani medium containing $100 \mu \mathrm{g} \mathrm{ml}^{-1}$ ampicillin and $100 \mu \mathrm{l}$ antifoam A concentrate (Sigma-Aldrich, catalogue number A5633) under continuous shaking until an $\mathrm{OD}_{600}$ of $0.8-1$. Protein expression was induced by adding $1 \mathrm{mM}$ isopropyl- $\beta$-D-thiogalactopyranoside and incubating for another $4 \mathrm{~h}$ at $37^{\circ} \mathrm{C}$. Refolding and purification were performed as previously described ${ }^{32}$. The His-tag was removed by the tobacco etch virus (TEV) protease cleavage after the refolding was completed. The TEV was removed during the subsequent cation exchange chromatography using a HiTrap SP FF column. The TEV cleavage was omitted for IFN $\lambda 3$. It is worth noting that the yield of IFN $\lambda 4$ protein is low with $1-2 \mathrm{mg}$ of purified protein from $121 \mathrm{E}$. coli culture. We did not note any consistent differences in the expression levels between IFN $\lambda 4$-P70 and IFN $\lambda$-S70.

Plasmids. The IFN $\lambda 4$-S70 constructs were generated by site-directed mutagenesis using PfuUltra II Fusion HS DNA polymerase according to the manufacturer's instructions (Agilent). For the mammalian cell expression, pEF2-IFN $\lambda 4$-S70-FLAG was generated on pEF2-IFN $\lambda 4$-P70-FLAG template using forward $5^{\prime}$-CCGCGGAG AATCCCTCCTGGGGC- $3^{\prime}$ and reverse $5^{\prime}$-GCCCCAGGAGGGATTCTCCGCGG- $3^{\prime}$ primers. For the expression in $E$. coli, pET15b-IFN $\lambda 4$-S70 was generated on pET15b-IFN $\lambda 4$-P70 construct using forward $5^{\prime}$-GCTGCTCGGACGAGGCGAAT CACGACGCGGACG-3' and reverse $5^{\prime}$-CGTCCGCGTCGTGATTCGCCTCGTC CGAGCAGC- $3^{\prime}$ primers. The following PCR programme was used: (1) $95^{\circ} \mathrm{C}$ for $30 \mathrm{~s}$; (2) $16 \mathrm{cycles}$ of $95^{\circ} \mathrm{C}$ for $30 \mathrm{~s}, 65^{\circ} \mathrm{C}$ for $1 \mathrm{~min}$ and $72^{\circ} \mathrm{C}$ for $4 \mathrm{~min}$; and (3) $72{ }^{\circ} \mathrm{C}$ for $10 \mathrm{~min}$

Cell culture. Unless otherwise stated, all cells were grown in Dulbecco's modified Eagle's medium (DMEM), which was supplemented with $10 \%$ fetal bovine serum, $100 \mathrm{U} \mathrm{ml}^{-1}$ penicillin and $100 \mu \mathrm{g} \mathrm{ml}^{-1}$ streptomycin. Cells were purchased from the German Collection of Microorganisms and Cell Cultures, DSMZ (HepG2 DSMZ-No.: ACC-180; HEK293 DSMZ-No.: ACC-305).

Activity assay in HEK293 cells. HEK293 cells were seeded at a density of $7.5 \times 10^{4}$ cells per well in a 48 -well plate and left to rest for $24 \mathrm{~h}$. After $24 \mathrm{~h}$, the cells were transfected with the pEF2 plasmid encoding IFN $\lambda$ R1, firefly luciferase under the control of the Mx1 promoter ${ }^{33}$ and Renilla luciferase under the control of the $\beta$-actin promoter. Eighteen hours post-transfection, cells were induced in quadruplicates with eight dilutions of IFN $\lambda 4$-P70 and IFN $\lambda 4$-S70 in the concentration range covering $0.006-100 \mathrm{ng} \mathrm{ml}^{-1}$ for $24 \mathrm{~h}$. After $24 \mathrm{~h}$, the cells were washed with PBS and lysed with Passive Lysis Buffer (Promega). The lysates were spun down at $10,000 \mathrm{~g}$ for $2 \mathrm{~min}$ at $4{ }^{\circ} \mathrm{C}$, and the cleared lysates were used for the measurement of luciferase activity (Dual-Luciferase Reporter Assay System, Promega). $\mathrm{EC}_{50}$, efficacy and exponential slope values were estimated in GraphPad prism version 5.04 by fitting a sigmoidal equation to the plot. The equation used for fitting was: firefly/Renilla ratio $=$ baseline $+($ efficacy - baseline $) /$ $\left(1+10^{\left(\left(\operatorname{logEC} C_{50}-X\right) \times \text { Slope }\right)}\right)$. Values for baseline were restrained to zero.

Real-time quantitative PCR. HepG2 cells were seeded at a density of $2 \times 10^{5}$ cells per well in 12-well plates, and after $24 \mathrm{~h}$, fresh media were added with the indicated IFNs in quadruplicates. After $4 \mathrm{~h}$ of incubation, cells were lysed and RNA was purified using an extraction kit (Omega) according to the manufacturer's instructions. Complementary DNA synthesis was performed with $1 \mu \mathrm{g}$ RNA using RevertAid reverse transcriptase and random hexamer primer according to the manufacturer's instructions (Thermo Scientific). The cDNA obtained from cells was quantified by real-time quantitative PCR using SYBR Green I (Roche) on Roche LightCycler 480 II. The following primers were used: GAPDH, forward $5^{\prime}$-CGACCACTTTGTCAAGCTCA- ${ }^{\prime}$ and reverse $5^{\prime}$-GGTGGTCCAGGGGTCTT ACT- $3^{\prime}$; IFIT1, forward $5^{\prime}$-CCTCCTTGGGTTCGTCTACA- $3^{\prime}$ and reverse $5^{\prime}$-GGC TGATATCTGGGTGCCTA- $3^{\prime}$; and $M X 1$, forward $5^{\prime}$-ACCTACAGCTGGCTCCT GAA- $3^{\prime}$ and reverse $5^{\prime}$-CGGCTAACGGATAAGCAGAG- $3^{\prime}$. The cycling parameters were: $95^{\circ} \mathrm{C}$ for $10 \mathrm{~min}$ followed by 45 cycles of $95^{\circ} \mathrm{C}$ for $10 \mathrm{~s}, 60^{\circ} \mathrm{C}$ for $20 \mathrm{~s}$ and $72{ }^{\circ} \mathrm{C}$ for $12 \mathrm{~s}$. The crossing points of the amplification curves were determined using the second derivate method on the Roche 480 LightCycler software 1.5 (Roche). The level of mRNA was normalized against the GAPDH mRNA content. The mean of the quadruplicates of the untreated control was used to calculate the fold induction for the other samples.

Antiviral assay. HepG2 cells were seeded at a concentration of $1.8 \times 10^{4}$ cells per well in 96-well plates and incubated with 20 dilutions of IFN $\lambda 4$-P70 or IFN $\lambda 4$-S70 
in the concentration range covering $0.008-200 \mathrm{ng} \mathrm{ml}^{-1}$ for $24 \mathrm{~h}$. The cells were then infected with EMCV (VR-129B strain) at a multiplicity of infection of 0.45 After $24 \mathrm{~h}$, MTT (3-(4,5-dimethylthiazol-2-yl)-2,5-diphenyl tetrazolium bromide; Sigma Aldrich) was added at a concentration of $0.8 \mathrm{mg} \mathrm{ml}^{-1}$ in DMEM, and the cells were incubated for additional $4 \mathrm{~h}$. The cells were dissolved in dimethyl sulfoxide (Sigma-Aldrich). MTT conversion was determined by measurement of the $A_{600}$. Measured absorptions in the IFN wells (Abs) were normalized as a percent of the cell control (CC) with the virus control (VC) subtracted, using the following equation: normalized cell survival $(\mathrm{NCS})=(\mathrm{Abs}-\mathrm{VC} /(\mathrm{CC}-\mathrm{VC})) \times 100$ to allow activity comparisons between plates. $\mathrm{EC}_{50}$, efficacy and exponential slope values were estimated as described in the section entitled 'Activity assay in HEK293 cells'.

Liver biopsies. Liver biopsies from patients $(n=104)$ were obtained in the outpatient clinic of the Division of Gastroenterology and Hepatology, University Hospital Basel, Switzerland. Parts of the biopsy material that were not needed for routine histopathology were used for research purposes after obtaining written informed consent. The use of biopsy material for this project was approved by the Ethikkommission Nordwest- und Zentralschweiz, Basel, Switzerland. Serum HCV RNA was quantified using the Cobas AmpliPrep/COBAS TaqMan HCV Test and the Cobas Amplicor Monitor from Roche Molecular Systems (Basel, Switzerland).

Total RNA and genomic DNA were isolated from liver biopsies using Trizol reagent (Invitrogen, Basel, Switzerland) and DNeasy Blood \& Tissue Kit (Qiagen, Hombrechtikon, Switzerland) according to the manufacturer's instructions. A fragment of 850 base pair covering $r s 368234815$ and $r s 117648444$ was amplified with the Expand High Fidelity PCR System (Roche) using forward 5'-ACTGTGTG TGCTGTGCCTTC- $3^{\prime}$ and reverse $5^{\prime}$-GGACGAGAGGGCGTTAGAG- $3^{\prime}$ primers. The product was purified on a column using NucleoSpin Gel and PCR clean-up (Macherey-Nagel AG, Oensingen, Switzerland) according to the manufacturer's instructions and then sequenced (Microsynth AG, Balgach, Switzerland).

pSVR was calculated using the 4-classifier genes as described previously ${ }^{10}$. Statistical analysis was performed using Prism6 (GraphPad software. La Jolla, CA, USA).

Genetic association study. Samples from patients with written informed consent for genetic studies and available DNA were collected from the Swiss Hepatitis C Cohort Study (SCCS), a multicenter study of HCV-infected patients enroled at eight major Swiss hospitals and their local affiliated centres, as described previously ${ }^{16,34}$, and from the Liver Unit of the Casa Sollievo della Sofferenza, San Giovanni Rotondo, Italy. The study was approved by the Ethics Committee of each SCCS center (Ethikkommission beider Basel EKBB (Basel), Ethikkommission des Kantons St Gallen (St Gallen), Comité intercantonal d'éthique (Neuchâtel), Comité d'éthique du département de Médecine (Geneva), Department Innere Medizin Ethisches Komitee (Zurich), Commission d'éthique de la recherche clinique (Lausanne), Comitato etico cantonale (Lugano)) and by the Ethical committee of 'Casa Sollievo della Sofferenza'. The study included Caucasian patients with spontaneous HCV clearance (defined as presence of anti-HCV antibodies but undetectable HCV RNA without previous antiviral treatment) and those who had chronic infection with HCV genotypes 1, 2, 3 or 4 and were assessable for response to therapy with pegIFN $\alpha$ and ribavirin, that is, who received $>80 \%$ of the recommended dose of each drug. Polymorphisms in the IFNL3/4 region ( $r s 12979860, r s 368234815, r s 117648444$ and $r s 8099917)$ were extracted from a GWAS-generated data set ${ }^{4}$ or genotyped by TaqMan (Applied Biosystems, Rotkreuz, Switzerland) or by the Competitive Allele Specific PCR (KASP) system (LGC Genomics, Herts, UK; http://www.lgcgenomics.com/kaspchallenge). Haplotypes were inferred using PHASE software, version 2.1 (University of Washington, available through Express Licensing Program, Seattle, WA, USA; http://stephenslab.uchicago.edu/software.html). Statistical analyses were performed using Stata (version 13.1, StataCorp LP, College Station, TX, USA). LD was assessed using the programs PWLD implemented in Stata. The association of polymorphisms with response to treatment and spontaneous clearance was performed by univariate and multivariate logistic regression. The multivariate models for spontaneous clearance are adjusted for age, sex and HCV risk. The multivariate models for response to treatment are adjusted age, sex, fibrosis stage and viral genotype.

\section{References}

1. Shepard, C. W., Finelli, L. \& Alter, M. J. Global epidemiology of hepatitis C virus infection. Lancet Infect. Dis. 5, 558-567 (2005).

2. Heim, M. H. 25 years of interferon-based treatment of chronic hepatitis C: an epoch coming to an end. Nat. Rev. Immunol. 13, 535-542 (2013).

3. Ge, D. et al. Genetic variation in IL28B predicts hepatitis C treatment-induced viral clearance. Nature 461, 399-401 (2009).

4. Rauch, A. et al. Genetic variation in IL28B is associated with chronic hepatitis C and treatment failure: a genome-wide association study. Gastroenterology 138, 1338-1345 (2010).

5. Suppiah, V. et al. IL28B is associated with response to chronic hepatitis C interferon-alpha and ribavirin therapy. Nat. Genet. 41, 1100-1104 (2009).
6. Tanaka, Y. et al. Genome-wide association of IL28B with response to pegylated interferon-alpha and ribavirin therapy for chronic hepatitis C. Nat. Genet. 41, 1105-1109 (2009).

7. Asselah, T. et al. Liver gene expression signature to predict response to pegylated interferon plus ribavirin combination therapy in patients with chronic hepatitis C. Gut 57, 516-524 (2008).

8. Chen, L. et al. Hepatic gene expression discriminates responders and nonresponders in treatment of chronic hepatitis $\mathrm{C}$ viral infection. Gastroenterology 128, 1437-1444 (2005).

9. Sarasin-Filipowicz, M. et al. Interferon signaling and treatment outcome in chronic hepatitis C. Proc. Natl Acad. Sci. USA 105, 7034-7039 (2008).

10. Dill, M. T. et al. Interferon-induced gene expression is a stronger predictor of treatment response than IL28B genotype in patients with hepatitis C. Gastroenterology 140, 1021-1031 (2011).

11. Honda, M. et al. Hepatic ISG expression is associated with genetic variation in interleukin 28B and the outcome of IFN therapy for chronic hepatitis C. Gastroenterology 139, 499-509 (2010).

12. Urban, T. J. et al. IL28B genotype is associated with differential expression of intrahepatic interferon-stimulated genes in patients with chronic hepatitis $\mathrm{C}$. Hepatology 52, 1888-1896 (2010).

13. Aka, P. V. et al. Association of the IFNL4-DeltaG allele with impaired spontaneous clearance of hepatitis C virus. J. Infect. Dis. 209, 350-354 (2014).

14. Thomas, D. L. et al. Genetic variation in IL28B and spontaneous clearance of hepatitis C virus. Nature 461, 798-801 (2009).

15. Prokunina-Olsson, L. et al. A variant upstream of IFNL3 (IL28B) creating a new interferon gene IFNL4 is associated with impaired clearance of hepatitis C virus. Nat. Genet. 45, 164-171 (2013).

16. Bibert, S. et al. IL28B expression depends on a novel TT/-G polymorphism which improves HCV clearance prediction. J. Exp. Med. 210, 1109-1116 (2013).

17. Hamming, O. J. et al. Interferon lambda 4 signals via the IFNlambda receptor to regulate antiviral activity against $\mathrm{HCV}$ and coronaviruses. $E M B O \mathrm{~J} . \mathbf{3 2}$, 3055-3065 (2013).

18. Smith, K. R. et al. Identification of improved IL28B SNPs and haplotypes for prediction of drug response in treatment of hepatitis $\mathrm{C}$ using massively paralle sequencing in a cross-sectional European cohort. Genome Med. 3, 57 (2011).

19. Gad, H. H. et al. Interferon-lambda is functionally an interferon but structurally related to the IL-10 family. J. Biol. Chem. 284, 20869-20875 (2009).

20. Miknis, Z. J. et al. Crystal structure of human interferon-lambda1 in complex with its high-affinity receptor interferon-lambdaR1. J. Mol. Biol. 404, 650-664 (2010).

21. Santantonio, T., Wiegand, J. \& Gerlach, J. T. Acute hepatitis C: current status and remaining challenges. J. Hepatol. 49, 625-633 (2008).

22. Francois-Newton, V. et al. USP18-based negative feedback control is induced by type I and type III interferons and specifically inactivates interferon alpha response. PLoS ONE 6, e22200 (2011).

23. Sarasin-Filipowicz, M. et al. Alpha interferon induces long-lasting refractoriness of JAK-STAT signaling in the mouse liver through induction of USP18/UBP43. Mol. Cell. Biol. 29, 4841-4851 (2009).

24. Jouvin-Marche, E. et al. Lymphocytes degranulation in liver in hepatitis $\mathrm{C}$ virus carriers is associated with IFNL4 polymorphisms and ALT levels. J. Infect. Dis. 209, 1907-1915 (2014).

25. Galmozzi, E. \& Aghemo, A. Nonsynonymous variant Pro70Ser (rs117648444) in IFNL4 gene identifies carriers of the rs368234815 DeltaG allele with higher HCV RNA decline during the first 4 weeks of pegylated interferon and ribavirin therapy in HCV-1 patients. J. Clin. Virol. 59, 274-275 (2014).

26. Ahlenstiel, G., Booth, D. R. \& George, J. Will IL28B polymorphisms remain relevant to direct-acting antiviral treatment paradigms? Antivir. Ther. 17, 1163-1170 (2012).

27. Holmes, J. A., Desmond, P. V. \& Thompson, A. J. Does IL28B genotyping still have a role in the era of direct-acting antiviral therapy for chronic hepatitis $C$ infection? J. Viral Hepat. 19, 677-684 (2012).

28. Muir, A. J. IL28B in the era of direct-acting antivirals for hepatitis C. J. Clin. Gastroenterol. 47, 222-227 (2013).

29. Zeuzem, S. et al. Faldaprevir and deleobuvir for HCV genotype 1 infection. New Engl. J. Med. 369, 630-639 (2013).

30. Meissner, E. G. et al. IFNL4-DeltaG genotype is associated with slower viral clearance in hepatitis $\mathrm{C}$, genotype-1 patients treated with sofosbuvir and ribavirin. J. Infect. Dis. 209, 1700-1704 (2014).

31. Bibert, S. et al. The IFNL3/4 [DELTA]G variant increases susceptibility to cytomegalovirus retinitis among HIV-infected patients. AIDS 28, 1885-1889 (2014).

32. Dellgren, C., Gad, H. H., Hamming, O. J., Melchjorsen, J. \& Hartmann, R. Human interferon-lambda 3 is a potent member of the type III interferon family. Genes Immun. 10, 125-131 (2009).

33. Jorns, C. et al. Rapid and simple detection of IFN-neutralizing antibodies in chronic hepatitis C non-responsive to IFN-alpha. J. Med. Virol. 78, 74-82 (2006). 
34. Prasad, L. et al. Cohort Profile: the Swiss Hepatitis C Cohort Study (SCCS). Int. J. Epidemiol. 36, 731-737 (2007).

\section{Acknowledgements}

We are grateful to Ole T. Hamming for his ideas and comments during the early stages of this project and to Sergei Kotenko for the gift of the pEF2-IFN $\lambda$ R1 plasmid. We are also grateful to Hans Henrik Gad and Lisbeth Heilesen for critical reading of this manuscript. The work was funded by the Danish Cancer Society (grant R20-A927, R.H.) and the Danish Council for Independent Research, Medical Research (grant 11-107588, R.H.). P.-Y.B. was supported by the Swiss National Foundation (324730-144054), the Leenaards Foundation and the Santos-Suarez Foundation. P.-Y.B. is a member of the HepaCute network (Collaborative Project) supported by the European Commission under the Health Cooperation Work Programme of the 7th Framework Programme (Grant agreement $n^{\circ}$ 260844). M.H.H. is supported by Swiss National Foundation (310030B_147089). Z.K. was supported by the Swiss National Science Foundation (31003A-143914) and the Leenaards Foundation. The Swiss Hepatitis C Cohort Study is supported by grants from the Swiss National Science Foundation (3347C0-108782/1 and 33CS30_148417/1). We thank all patients from the Swiss Hepatitis C Cohort Study, as well as collaborators from the clinical, laboratory and data centres and all study nurses.

\section{Author contributions}

E.T.-D., S.B., F.H.T.D., I.K., S.J. and E.C. performed the experiments and analysed the data. M.H.H., P.-Y.B. and R.H. supervised the study and analysed the data. E.T.-D., S.B., F.H.T.D., M.H.H., P.-Y.B. and R.H. wrote the paper with input from the remaining of the authors. Z.K. provided statistical support. V.A., A.C., L.K., R.M., A.M., D.M., B.M., F.N., R.S., D.S. and N.S. collected or processed clinical data and/or samples.

\section{Additional information}

Supplementary Information accompanies this paper at http://www.nature.com/ naturecommunications

Competing financial interests: The authors have no conflicting financial interests.

Reprints and permission information is available online at http://npg.nature.com/ reprintsandpermissions/

How to cite this article: Terczyńska-Dyla, E. et al. Reduced IFN $\lambda 4$ activity is associated with improved HCV clearance and reduced expression of interferon-stimulated genes. Nat. Commun. 5:5699 doi: 10.1038/ncomms6699 (2014).

\section{Members of the Swiss Hepatitis C Cohort Study Group}

Laura Rubbia-Brandt ${ }^{17}$, Gladys Martinetti ${ }^{18}$, Meri Gorgievski ${ }^{19}$, Jean-François Dufour ${ }^{20}$, Hans Hirsch ${ }^{21}$, Beat Helbling 22,23 , Stephan Regenass ${ }^{24}$, Guenter Dollenmaier ${ }^{25}$, Gieri Cathomas ${ }^{26}$

${ }^{17}$ Service de Pathologie Clinique, Hôpital Cantonal, Rue Michel-Servet 1, 1206 Genève, Switzerland. ${ }^{18}$ Servizio di Microbiologia, EOLAB, 6500 Bellinzona, Switzerland. ${ }^{19}$ Fachbereich Virologie/Molekularbiologie, Friedbühlstrasse 51, 3010 Bern, Switzerland. 20 University Clinic Visceral Surgery and Medicine, Inselspital, Freiburgstrasse, 3010 Bern, Switzerland. ${ }^{21}$ Institut für Med. Mikrobiologie, der Universität Basel, Petersplatz 10, 4003 Basel, Switzerland. ${ }^{22}$ Division of Gastroenterology and Hepatology, Stadtspital Waid, Zurich, Switzerland. ${ }^{23}$ Gastroenterologie Hepatologie Bethanien; Toblerstrasse 51, 8044 Zürich, Switzerland. ${ }^{24}$ Abt. für Klin. Immunologie, Universitätsspital, Häldeliweg 4, 8044 Zürich, Switzerland. ${ }^{25}$ Institut für klin.Mikrobiol. u. Immunologie, Frohbergstr. 3, 9001 St. Gallen, Switzerland. ${ }^{26}$ Kantonales Institut für Pathologie, Rheinstrasse 37, 4410 Liestal, Switzerland 


\section{Corrigendum: Reduced IFN $\lambda 4$ activity is associated with improved HCV clearance and reduced expression of interferon-stimulated genes}

Ewa Terczyńska-Dyla, Stephanie Bibert, Francois H. T. Duong, Ilona Krol, Sanne Jørgensen, Emilie Collinet, Zoltán Kutalik, Vincent Aubert, Andreas Cerny, Laurent Kaiser, Raffaele Malinverni, Alessandra Mangia, Darius Moradpour, Beat Müllhaupt, Francesco Negro, Rosanna Santoro, David Semela, Nasser Semmo, Swiss Hepatitis C Cohort Study Group, Markus H. Heim, Pierre-Yves Bochud \& Rune Hartmann

Nature Communications 5:5699 doi: 10.1038/ncomms6699 (2014); Published 23 Dec 2014; Updated 29 Jun 2015

In this Article, Rune Hartman was omitted as joint supervisor of this work. The correct list of joint supervisors is: Markus H. Heim, Pierre-Yves Bochud and Rune Hartmann. 